\title{
Evaluation of A 55-Gene Classifier As A Prognostic Biomarker for Adjuvant Chemotherapy in Stage III Colon Cancer Patients
}

Eiji Oki ( $\sim$ okieiji@surg2.med.kyushu-u.ac.jp )

Kyushu University

\section{Eiji Shinto}

National Defense Medical College

Mototsugu Shimokawa

National Hospital Organization Kyushu Cancer Center

Shigeki Yamaguchi

Saitama Medical University International Medical Center

Megumi Ishiguro

Tokyo Medical and Dental University

Seiji Hasegawa

Saiseikai Yokohamashi Nanbu Hospital

Yasumasa Takii

Niigata Cancer Center Hospital

Hideyuki Ishida

Saitama Medical University

Tetsuya Kusumoto

National Hospital Organization Kyushu Medical Center

Masaru Morita

National Hospital Organization Kyushu Cancer Center

Naohiro Tomita

Hyogo College of Medicine

Manabu Shiozawa

Kanagawa Cancer Center

Masafumi Tanaka

Takano Hospital

\section{Heita Ozawa}

Tochigi Cancer Center

\section{Yojiro Hashiguchi}

Teikyo University School of Medicine

Shinobu Ohnuma 
Tohoku University Hospital

\section{Sachiyo Tada}

LS Business, Sysmex Corporation

\section{Tomoko Matsushima}

LS Business, Sysmex Corporation

\section{Kazuo Hase}

National Defense Medical College

\section{Research Article}

Keywords: Colon cancer, predictive, adjuvant chemotherapy, oxaliplatin, subtyping

Posted Date: June 17th, 2021

DOl: https://doi.org/10.21203/rs.3.rs-579197/v1

License: (c) (i) This work is licensed under a Creative Commons Attribution 4.0 International License. Read Full License

Version of Record: A version of this preprint was published at BMC Cancer on December 1st, 2021. See the published version at https://doi.org/10.1186/s12885-021-09088-6. 


\section{Abstract \\ Background}

Adjuvant chemotherapy reduces the recurrence risk in stage III colon cancer (CC). However, better prognostic and predictive biomarkers are required to stratify patients for treatment. We constructed a 55gene classifier (55GC) and investigated its utility for classifying patients with stage III CC.

\section{Methods}

We retrospectively identified patients with stage III CC aged 20-79 years who received adjuvant chemotherapy with or without oxaliplatin during 2009-2012.

\section{Results}

Among 938 eligible patients, 203 and 201 patients who received adjuvant chemotherapy with and without oxaliplatin, respectively, were selected by propensity-score matching. Of these, 95 patients from each group were analyzed and their 5-year relapse-free survival (RFS) rates with and without oxaliplatin were $73.7 \%$ and $77.1 \%$, respectively. The hazard ratios for 5 -year RFS for adjuvant chemotherapy (fluoropyrimidine), with or without oxaliplatin, were $1.241(95 \% \mathrm{Cl}, 0.465-3.308 ; P=0.67$ ) and 0.791 (95\% $\mathrm{Cl}, 0.329-1.901 ; P=0.60)$, respectively. Stratification using the 55GC revealed that $52(27.3 \%), 78(41.1 \%)$, and $60(31.6 \%)$ patients had microsatellite instability (MSI)-like, chromosomal instability (CIN)-like, and stromal subtypes, respectively. The 5-year RFS rates were $84.3 \%$ and $72.0 \%$ in patients treated with and without oxaliplatin, respectively, for the MSI-like subtype (HR, $0.495 ; 95 \% \mathrm{Cl}, 0.145-1.692 ; P=0.25)$. No RFS rate differences were noted in CIN-like or stromal subtypes. Stratification by cancer sidedness for each subtype showed improved RFS only in left-sided primary cancer treated with oxaliplatin for the MSIlike subtype $(P=0.007)$. The 5-year RFS rates for the MSI-like subtype in left-sided cancer were $100 \%$ and $53.9 \%$ with and without oxaliplatin, respectively.

\section{Conclusions}

Subclassification using 55GC and tumor sidedness revealed increased RFS of patients within the MSI-like subtype with stage III left-sided CC treated with fluoropyrimidine with oxaliplatin relative to those treated without oxaliplatin. However, the predictive power of 55GC subtyping alone did not reach statistical significance in this cohort, warranting larger prospective studies.

\section{Trial registration:}


The study protocol was registered in the University Hospital Medical Education Network (UMIN) clinical trial registry (UMIN study ID 000023879).

\section{Background}

Colorectal cancer remains one of the most common causes of cancer-related deaths worldwide [1]. Adjuvant chemotherapy in stage III colon cancer (CC) after curative-intent resection prolongs survival and reduces the risk of recurrence [2]. Pivotal trials have shown superior outcomes for fluoropyrimidine in combination with oxaliplatin compared with fluoropyrimidine alone in most patient populations; however, the evidence is less well established in elderly patients. Emerging data have also led to debate over the optimal duration of chemotherapy, specifically in the context of increased toxicity [3]. Furthermore, subclassification of stage III CC is an ongoing process with revision based on the accumulating patient survival data and cancer presentation [4]. Therefore, better prognostic and predictive biomarkers are required to stratify patients for adjuvant therapies based on the chemotherapy regimen and duration.

The consensus molecular subtypes (CMS) are robust classification systems for colorectal cancer that include over 600 genes. DNA microarray analysis of CMS can provide prognostic information [5]. CMS is also potentially predictive, with different sensitivities to adjuvant chemotherapy. We previously simplified this classification by constructing a 55-gene classifier (55GC) focusing on genes located on the long arms of chromosomes 18 and 20 as well as stroma-related genes [6]. Using the 55GC, we categorized stage II/III CC into three subtypes with different recurrence rates: "microsatellite instability (MSI)-like," "chromosomal instability (CIN)-like," and "stromal" subtypes and showed prognostic utility in a single institutional study. We conducted a validation study by the 55-gene classifier for assessing stratification recurrence (55 STAR) risk. Further, 55GC-based subtyping was able to stratify stage II CC recurrence risk in a multi-institutional validation cohort study of 232 patients [7]. To expand on these previous findings, here we hypothesized that $55 \mathrm{GC}$ could be utilized to stratify survival of patients with stage III CC that received adjuvant chemotherapy with or without oxaliplatin.

\section{Materials And Methods}

\section{Tissue samples}

We retrospectively identified consecutive patients with stage III colon and rectosigmoid CC aged 20-79 years who underwent curative surgery (R0) and received adjuvant chemotherapy with or without oxaliplatin from 15 institutions in Japan between January 1, 2009 and December 31, 2012. Samples from patients who received neoadjuvant treatment, had multiple active cancers, died, or had recurrence within 60 days post-surgery were excluded from the study. Patient characteristics were recorded. The study protocol was approved by the institutional review boards of Kyushu University (study ID 28-69), National Defense Medical College (study ID 2477), Saitama Medical University International Medical Center (study ID 16-051), Tokyo Medical and Dental University (study ID G2016-007), Saiseikai Yokohamashi Nanbu Hospital (study ID 2017-D21), Niigata Cancer Center Hospital (study ID 796), 
Saitama Medical University Saitama Medical Center (study ID 1812), National Hospital Organization Kyushu Medical Center (study ID 16C058), National Hospital Organization Kyushu Cancer Center (study ID 2016-48), Hyogo College of Medicine (study ID Hi326), Kanagawa Cancer Center (study ID 2017-8), Takano Hospital (study ID 16 - 04), Tochigi Cancer Center (study ID A432), Teikyo University School of Medicine (study ID 16-057), Tohoku University (study ID 2016-1-222) and Sysmex Corporation (study ID 2015-71), and was registered in the University Hospital Medical Education Network Clinical Trial Registry (UMIN study ID 000023879). All of the methods were carried out in accordance with relevant guidelines and regulations. Because this study was a retrospective observational study carried out in Japan, informed consent was obtained using the opt-out/opt-in approach according to each participating institution's policy (as per Japanese ethical guidelines for observational study, consent of the family is not required for dead participants). The Consolidated Standards of Reporting Trials (CONSORT) diagram is shown in Fig. 1.

\section{Gene expression analysis}

Formalin-fixed paraffin-embedded (FFPE) primary cancer tissue specimens containing the tumor invasive front with the greatest depth of invasion were collected from each institution, and a single 5 - $\mu \mathrm{m}$ section was sent to Takeda Pathology Center (Osaka, Japan) for assessment. Total RNA was extracted from fewer than four unstained 10- $\mu \mathrm{m}$ sections for gene expression microarray assay profiling using the RNeasy FFPE Kit (Qiagen; Valencia, CA). Samples with insufficient RNA quality for microarray analysis were omitted from the cohort. Gene expression data were generated using the Affymetrix GeneChip Human Genome U133 Plus 2.0 Array (Thermo Fisher Scientific; Waltham, MA) and analyzed with the 55GC model as described previously [6]. DNA was extracted using the QIAamp DNA FFPE Tissue Kit (Qiagen). RAS mutations were assessed using the MEBGEN RASKET KIT (Medical \& Biological Laboratories; Nagoya, Japan).

\section{Statistical analyses}

The primary endpoint was RFS, defined as the time from surgery to first CC recurrence or death from any cause. A propensity score method was used to reduce selection bias, and a logistic regression model was used to calculate patient propensity scores. Propensity score matching was performed for the number of lymph node metastases, tumor location, sex, and age in a 1:1 ratio using a caliper width of 0.1 .

Demographic characteristics are summarized using contingency tables. The RFS curve was estimated using the Kaplan-Meier method and compared between groups using log-rank tests. HRs and $95 \% \mathrm{Cls}$ were calculated using a Cox proportional hazards model. Risk factors for RFS were assessed using a Cox proportional hazards model with a backward elimination method that included known clinicopathological prognostic factors and gene mutations as covariates. Subgroup analysis was performed for age ( $<70$ vs. $\geq 70$ years), sex (male vs. female), carcinoembryonic antigen (< upper limit of normal [ULN] vs. $\geq U L N$ ), tumor location (left vs. right), T stage (T1-T3 vs. T4), lymph node metastasis (N1 vs. N2-N3), tumor grade (poorly differentiated and mucinous adenocarcinoma vs. tubular adenocarcinoma), vascular invasion (v0 vs. v1-v3), subtype (CIN vs. MSI vs. stromal), and RAS (wild vs. mutant). Fisher's exact test was used to compare patient characteristics between groups. $P$ values were two-sided, and $P<0.05$ was 
considered statistically significant. All statistical analyses were performed using the Statistical Analysis System (SAS) version 9.4 (SAS Institute, Cary, NC).

\section{Results}

\section{Patient characteristics}

Among 938 eligible patients, 203 and 201 patients receiving adjuvant chemotherapy with and without oxaliplatin, respectively, were selected using propensity score matching. After excluding patients with lowquality specimens and those who had received chemotherapy for $<3$ months, 95 patients from each group were analyzed (Fig. 1). In the overall cohort of 190 patients (Table 1), 98 (51.6\%) patients were men, 146 (76.8\%) were aged < 70 years, and $126(66.3 \%)$ had left-sided tumors. Regarding histopathological characteristics, there were more patients with $\mathrm{T} 4$ stage cancer in the cohort treated with oxaliplatin ( $44 / 95$ patients, $46.3 \%)$ than in the cohort treated without oxaliplatin (34/95 patients, $35.8 \%)$ $(P=0.022)$. In addition, there were more patients with $<12$ resected lymph nodes in the cohort treated with oxaliplatin $(15 / 95$ patients, $15.8 \%)$ than in the cohort treated without oxaliplatin (5/95 patients, $5.3 \%)(P=0.018)$. The 5 -year RFS rates were $73.7 \%$ and $77.1 \%$ in patients treated without and with oxaliplatin, respectively (Fig. 2; hazard ratio [HR]: 0.858; 95\% confidence interval [Cl]: 0.484-1.522). 
Table 1

Patient characteristics

\begin{tabular}{|c|c|c|c|c|c|}
\hline \multirow[t]{2}{*}{ Factors } & & $\begin{array}{l}\text { Oxaliplatin }(-)(N= \\
95)\end{array}$ & $\begin{array}{l}\text { Oxaliplatin } \\
(+) \\
(N=95)\end{array}$ & $\begin{array}{l}\text { Total } \\
(N= \\
190)\end{array}$ & \multirow{2}{*}{$\begin{array}{l}P \\
\text { value }\end{array}$} \\
\hline & & $\mathrm{n}(\%)$ & $\mathrm{n}(\%)$ & $\mathrm{n}(\%)$ & \\
\hline \multirow[t]{2}{*}{ Sex } & Male & $46(48.4)$ & $52(54.7)$ & $\begin{array}{l}98 \\
(51.6)\end{array}$ & 0.3838 \\
\hline & Female & $49(51.6)$ & $43(45.3)$ & $\begin{array}{l}92 \\
(48.4)\end{array}$ & \\
\hline \multirow[t]{2}{*}{ Age (years) } & $<70$ & $71(74.7)$ & 75 (78.9) & $\begin{array}{l}146 \\
(76.8)\end{array}$ & 0.4915 \\
\hline & $\geq 70$ & $24(25.3)$ & $20(21.1)$ & $\begin{array}{l}44 \\
(23.2)\end{array}$ & \\
\hline \multirow[t]{3}{*}{ CEA } & $<U L N$ & $63(66.3)$ & $50(52.6)$ & $\begin{array}{l}113 \\
(59.5)\end{array}$ & 0.6105 \\
\hline & $\geq U L N$ & $31(32.6)$ & $20(21.1)$ & $\begin{array}{l}51 \\
(26.8)\end{array}$ & \\
\hline & Unknown & $1(1.1)$ & $25(26.3)$ & $\begin{array}{l}26 \\
(13.7)\end{array}$ & \\
\hline \multirow[t]{2}{*}{ Tumor location } & $\begin{array}{l}\text { Right } \\
\text { side }\end{array}$ & $31(32.6)$ & $33(34.7)$ & $\begin{array}{l}64 \\
(33.7)\end{array}$ & 0.7588 \\
\hline & Left side & $64(67.4)$ & $62(65.3)$ & $\begin{array}{l}126 \\
(66.3)\end{array}$ & \\
\hline \multirow[t]{2}{*}{ T stage } & T1-T3 & $61(64.2)$ & $51(53.7)$ & $\begin{array}{l}112 \\
(58.9)\end{array}$ & 0.1403 \\
\hline & $\mathrm{T} 4$ & $34(35.8)$ & $44(46.3)$ & $\begin{array}{l}78 \\
(41.1)\end{array}$ & \\
\hline \multirow[t]{2}{*}{ Tumor grade } & $\begin{array}{l}\text { por \& } \\
\text { muc }\end{array}$ & $4(4.2)$ & $13(13.7)$ & $17(8.9)$ & 0.0222 \\
\hline & tub & $91(95.8)$ & $82(86.3)$ & $\begin{array}{l}173 \\
(91.1)\end{array}$ & \\
\hline \multirow[t]{2}{*}{ Lymphatic invasion } & Negative & $29(30.5)$ & $26(27.4)$ & $\begin{array}{l}55 \\
(28.9)\end{array}$ & 0.6313 \\
\hline & Positive & $66(69.5)$ & $69(72.6)$ & $\begin{array}{l}135 \\
(71.1)\end{array}$ & \\
\hline
\end{tabular}




\begin{tabular}{|c|c|c|c|c|c|}
\hline \multicolumn{2}{|l|}{ Factors } & \multirow{2}{*}{$\begin{array}{l}\text { Oxaliplatin }(-)(N= \\
95) \\
\text { n (\%) }\end{array}$} & \multirow{2}{*}{$\begin{array}{l}\text { Oxaliplatin } \\
(+) \\
(N=95) \\
n(\%)\end{array}$} & \multirow{2}{*}{$\begin{array}{l}\text { Total } \\
(N= \\
190) \\
\mathrm{n}(\%)\end{array}$} & \multirow{2}{*}{$\begin{array}{l}P \\
\text { value }\end{array}$} \\
\hline & & & & & \\
\hline \multirow[t]{2}{*}{ Vascular invasion } & Negative & $28(29.5)$ & $23(24.2)$ & $\begin{array}{l}51 \\
(26.8)\end{array}$ & 0.4130 \\
\hline & Positive & $67(70.5)$ & $72(75.8)$ & $\begin{array}{l}139 \\
(73.2)\end{array}$ & \\
\hline \multirow[t]{3}{*}{$\mathrm{N}$ stage } & N1 & $39(41.1)$ & $36(37.9)$ & $\begin{array}{l}75 \\
(39.5)\end{array}$ & 0.0940 \\
\hline & N2 & $54(56.8)$ & $50(52.6)$ & $\begin{array}{l}104 \\
(54.7)\end{array}$ & \\
\hline & N3 & $2(2.1)$ & $9(9.5)$ & $11(5.8)$ & \\
\hline \multirow[t]{2}{*}{$\begin{array}{l}\text { Number of resected lymph } \\
\text { nodes }\end{array}$} & $<12$ & $5(5.3)$ & $15(15.8)$ & $\begin{array}{l}20 \\
(10.5)\end{array}$ & 0.0181 \\
\hline & $\geq 12$ & $90(94.7)$ & $80(84.2)$ & $\begin{array}{l}170 \\
(89.5)\end{array}$ & \\
\hline
\end{tabular}

\section{5-gene classifier subtype analysis}

Of the total cohort, 55GC analysis revealed $52(27.3 \%)$ patients with a MSI-like subtype, 78 (41.1\%) patients with a CIN-like subtype, and $60(31.6 \%)$ patients with a stromal subtype. The patient characteristics within each subtype are shown in Supplementary Table S1. MSI-like subtype tumors were more likely to be right-sided, whereas CIN-like subtype tumors were more likely to be left-sided compared with the overall cohort. All (100\%) CIN-like subtype tumors had non-mucinous histology compared with $95.8 \%$ in the overall cohort. MSI-like subtype tumors had higher proportions of N2 (34/52 tumors, $65.4 \%$ ) and N3 (5/52 tumors, 9.6\%) stages compared with the overall cohort.

\section{Survival analysis according to 55-gene classifier analysis and chemotherapy regimen}

Comparisons of RFS in patients treated with and without oxaliplatin according to the 55GC subtype are shown in Fig. 3. The 5-year RFS rates were $84.3 \%$ and $72.0 \%$ in patients treated with and without oxaliplatin, respectively, for the MSI-like subtype (HR, 0.495; $95 \% \mathrm{Cl}, 0.145-1.692)$; however, the trend was not statistically significant (log-rank $P=0.25$ ). There was no difference in RFS in CIN-like subtype patients according to oxaliplatin treatment ( $\mathrm{HR}, 1.241 ; 95 \% \mathrm{Cl}, 0.465-3.308$; log-rank $P=0.67)$. RFS was also unchanged in the stromal subtype patients regardless of oxaliplatin treatment $(\mathrm{HR}, 0.791 ; 95 \% \mathrm{Cl}, 0.329-$ $1.901 ; \log$-rank $P=0.60)$. Further subdivision into left- and right-sided primary cancer of the subtypes 
showed improved RFS only in left-sided primary cancer of the MSI-like subtype treated with oxaliplatin (Fig. 4; log-rank $P=0.0071$ ). The 5-year RFS rates for the MSI-like subtype in left-sided cancer were $100 \%$ and $53.9 \%$ with and without oxaliplatin, respectively. No significant differences in RFS were noted between subtypes when stratified by treatment with (log-rank $P=0.23$ ) and without oxaliplatin (log-rank $P=0.37$; Supplementary Fig. S1).

Subgroup analysis is shown in Supplementary Fig. S2. Comparison of RFS in patients treated with or without oxaliplatin revealed no significant differences according to patient characteristics (age, sex) or histopathological findings (tumor location, $\mathrm{T}$ stage, $\mathrm{N}$ stage, tumor grade, and vascular invasion).

\section{Discussion}

Despite significant progress in the development of prognostic and predictive biomarkers in CC, particularly RAS mutation status and deficient mismatch repair (dMMR) status to guide therapy for metastases [8, 9], there is an ongoing need for progress in the molecular analysis of early stage CC to guide adjuvant therapy. While dMMR status may indicate a lack of efficacy of fluoropyrimidine-only regimens without oxaliplatin, there are no other validated predictive tumor biomarkers in early stage CC [10]. Several multigene expression profiling systems such as Oncotype DX (Genomic Health; Redwood City, CA) and ColoPrint (Agendia; Amsterdam, Netherlands), have been developed [11]. However, they are not subtyping systems; therefore, they have prognostic but no predictive value for recurrence risk. In contrast, the predictive potential of molecular subtypes in CC has been demonstrated recently in prospective trials [12-14]. While the classification of CMS is considered the most robust classification based on comprehensive gene expression profiling [5], other classifications have been developed and validated [15-18]. However, there is debate over the optimal method of identification of subtypes and difficulties in the practical widespread measurement of these genotypes in routine clinical practice [19]. Recently, other gene set classifiers obtained from microarray used in CMS were reported. Similar prognostic utility was shown using 99 or 200 gene sets [20]. In this study, we suggest the possible utility of a 55 gene set, especially when paired with other cancer properties such as sidedness, which may provide potentially prognostic and predictive information for adjuvant therapy in early stage CC after curative-intent resection.

Post-hoc analyses of tumor tissue from patients in large randomized trials of adjuvant chemotherapy have revealed the overall poor prognosis of certain molecular subtypes. For example, analysis of the National Surgical Adjuvant Breast and Bowel Project (NSABP) C-07 clinical trial demonstrated poor prognosis in both stage II and III patients with a 'stem-like' subtype identified from three different subtyping methods [21]. This is consistent with our findings herein, which show a tendency for poorer prognosis of the stromal subtype in stage III patients as well as our previous results in stage II/III CC patients [6]. Furthermore, in the aforementioned NSABP C-07 retrospective analysis, the stem-like subtype from Colorectal Cancer Assigner classification (CRCA) predicted a lack of benefit for the addition of oxaliplatin [21]. Similar to the NSABP C-07 study, our cohort demonstrated a 3.4\% improvement in 5-year RFS with the addition of oxaliplatin to adjuvant chemotherapy. Considering this result, the potential 
additive benefit of oxaliplatin for adjuvant chemotherapy in the MSI-like subtype from our 55GC system requires further investigation for validation. Similar to the CMS classification, the 55GC system requires caution; the MSI-like subtype is not identical to MSI-high or dMMR CC.

Our study has the following major limitation: the relatively small number of patients in each subgroup, especially after accounting for propensity score matching. Nevertheless, the results suggest the potential benefit of adjuvant chemotherapy with oxaliplatin in the MSI-like subtype, when accounting for tumor sidedness. These findings require further prospective validation in an independent cohort to determine the true clinical significance. Combined with deeper analysis of genomic and histopathologic correlates, including the immune and tumor microenvironment, this would enhance our understanding of the biological underpinnings of each subtype and their predictive efficacy.

\section{Conclusions}

In conclusion, the current 55GC study illustrated that oxaliplatin may have an additive effect in adjuvant chemotherapy for the MSI-like subtype for left-sided primary CC. Hence, future study with a larger number of CC cases are warranted.

\section{Abbreviations}

\section{$55 \mathrm{GC}$}

55 gene classifier

CC

Colon cancer

CEA

Carcinoembryonic antigen

Cl

Confidence interval

CIN

Chromosomal instability

CMS

Consensus molecular subtypes

\section{CONSORT}

Consolidated Standards of Reporting Trials

\section{CRCA}

Colorectal Cancer Assigner

\section{DNA}

Deoxyribonucleic acid

FFPE

Formalin-fixed paraffin-embedded

HR 
Hazard ratio

MSI

Microsatellite instability

NSABP

National Surgical Adjuvant Breast and Bowel Project

$\mathrm{P}$

P-value

RFS

Relapse-free survival

RNA

Ribonucleic acid

STAR

Stratification recurrence

ULN

Upper limit of normal

UMIN

University Hospital Medical Education Network

\section{Declarations}

\section{ETHICS APPROVAL AND CONSENT TO PARTICIPATE}

The study protocol was approved by the following institutional review boards: Kyushu University (study ID 28-69), National Defense Medical College (study ID 2477), Saitama Medical University International Medical Center (study ID 16-051), Tokyo Medical and Dental University (study ID G2016-007), Saiseikai Yokohamashi Nanbu Hospital (study ID 2017-D21), Niigata Cancer Center Hospital (study ID 796), Saitama Medical University Saitama Medical Center (study ID 1812), National Hospital Organization Kyushu Medical Center (study ID 16C058), National Hospital Organization Kyushu Cancer Center (study ID 2016-48), Hyogo College of Medicine (study ID Hi326), Kanagawa Cancer Center (study ID 2017-8), Takano Hospital (study ID 16-04), Tochigi Cancer Center (study ID A432), Teikyo University School of Medicine (study ID 16-057), Tohoku University (study ID 2016-1-222) and Sysmex Corporation (study ID 2015-71). All methods were carried out in accordance with relevant guidelines and regulations. Because this study was a retrospective observational study carried out in Japan, informed consent was obtained using the opt-out/opt-in approach according to each participating institution's policy (as per Japanese ethical guidelines for observational study, consent of the family is not required for dead participants).

\section{CONSENT FOR PUBLICATION}

Not applicable.

\section{AVAILABILITY OF DATA AND MATERIALS}


The cDNA microarray datasets generated during the current study are available from the corresponding author upon reasonable request. Repository to Gene Expression Omnibus (GEO) database are not prepared by concern of the Japanese Act on the Protection of Personal Information.

\section{COMPETING INTEREST}

E.O. has been part of a speakers' bureau at Bayer, Chugai Pharmaceutical, Eli Lilly, Merck Serono, Ono Pharmaceutical, Taiho Pharmaceutical, Takeda Pharmaceutical, and Yakult Honsha. M.S. has a consulting/advisory role with Sysmex. S.Y. has been part of a speakers' bureau at Chugai Pharmaceutical, Covidien, Eli Lilly, Merck Serono, Johnson \& Johnson, and Taiho Pharmaceutical. M.I. has received honoraria from Merck Serono, Taiho Pharmaceutical, and Yakult Honsha, and has a consulting/advisory role with Taiho Pharmaceutical and Yakult Honsha. N.T. has received research funding from Chugai Pharmaceutical and Taiho Pharmaceutical. Y.H. has received honoraria from Bayer, Chugai Pharmaceutical, Kaken Pharmaceutical, Merck Serono, Sanofi, Taiho Pharmaceutical, and Takeda Pharmaceutical, and has received research funding from Chugai Pharmaceutical, Kaken Pharmaceutical, Merck Serono, Sanofi, Taiho Pharmaceutical, and Takeda Pharmaceutical. S.T. and T.M. are employed at Sysmex and own stock in Sysmex. E.S., S.H., Y.T., H.I., T.K., M.M, M.S., M.T., H.O., S.O., and K.H. have no conflicts of interest to disclose.

\section{FUNDING}

This work was supported by Sysmex Corporation. The funding source had no involvement in study design; in the collection, analysis, and interpretation of data; in the writing of the report; or in the decision to submit the article for publication.

\section{AUTHOR'S CONTRIBUTIONS}

S.T. and T.M.: Quality control of data and algorithms. E.O., E.S., M.S., S.T., T.M., K.H.: Data analysis and interpretation. M.S.: Statistical analysis. E.O., E.S., M.S., S.T., T.M., K.H.: Manuscript preparation. E.O., E.S., S.Y., M.I., S.H., Y.T., H.I., T.K., M.M., N.T., Ma.S., M.T., H.O., Y.H., S.O., S.T., T.M., K.H.: Study concepts, Study design, Data acquisition, Manuscript editing, and Manuscript review.

\section{ACKNOWLEDGMENTS}

Editorial support, in the form of medical writing, assembling tables, and creating high-resolution images based on authors' detailed directions, collating author comments, copyediting, fact-checking, and referencing, was provided by Editage, Cactus Communications, and was funded by Sysmex Corporation.

\section{References}

1. Arnold M, Sierra MS, Laversanne M, Soerjomataram I, Jemal A, Bray F. Global patterns and trends in colorectal cancer incidence and mortality. Gut. 2017;66(4):683-91. 
2. André T, Boni C, Navarro M, Tabernero J, Hickish T, Topham C, et al. Improved overall survival with oxaliplatin, fluorouracil, and leucovorin as adjuvant treatment in stage II or III colon cancer in the MOSAIC trial. J Clin Oncol. 2009;27(19):3109-16.

3. Grothey A, Sobrero AF, Shields AF, Yoshino T, Paul J, Taieb J, et al. Duration of adjuvant chemotherapy for stage III colon cancer. N Engl J Med. 2018;378(13):1177-88.

4. Gunderson LL, Jessup JM, Sargent DJ, Greene FL, Stewart AK. Revised TN categorization for colon cancer based on national survival outcomes data. J Clin Oncol. 2010;28(2):264-71.

5. Guinney J, Dienstmann R, Wang X, de Reyniès A, Schlicker A, Soneson C, et al. The consensus molecular subtypes of colorectal cancer. Nat Med. 2015;21(11):1350-6.

6. Gotoh K, Shinto E, Yoshida Y, Ueno H, Kajiwara Y, Yamadera M, et al. Prognostic model of stage II/III colon cancer constructed using gene expression subtypes and KRAS mutation status. J Clin Exp Oncol [Internet]. 2018;07(02). Available from: http://dx.doi.org/10.4172/2324-9110.1000214

7. Shinto E, Oki E, Shimokawa M, Yamaguchi S, Ishiguro M, Morita M, et al. A validation study for recurrence risk stratification of stage II colon cancer using the 55-gene classifier. Oncology. 2020;98(8):534-41.

8. Karapetis CS, Khambata-Ford S, Jonker DJ, O'Callaghan CJ, Tu D, Tebbutt NC, et al. K-ras mutations and benefit from cetuximab in advanced colorectal cancer. N Engl J Med. 2008;359(17):1757-65.

9. Le DT, Durham JN, Smith KN, Wang H, Bartlett BR, Aulakh LK, et al. Mismatch repair deficiency predicts response of solid tumors to PD-1 blockade. Science. 2017;357(6349):409-13.

10. Sargent DJ, Marsoni S, Monges G, Thibodeau SN, Labianca R, Hamilton SR, et al. Defective mismatch repair as a predictive marker for lack of efficacy of fluorouracil-based adjuvant therapy in colon cancer. J Clin Oncol. 2010;28(20):3219-26.

11. Sharif S, O'Connell MJ. Gene signatures in stage II colon cancer: A clinical review. Curr Colorectal Cancer Rep. 2012;8(3):225-31.

12. Mooi JK, Wirapati P, Asher R, Lee CK, Savas P, Price TJ, et al. The prognostic impact of consensus molecular subtypes (CMS) and its predictive effects for bevacizumab benefit in metastatic colorectal cancer: molecular analysis of the AGITG MAX clinical trial. Ann Oncol. 2018;29(11):2240-6.

13. Lenz H-J, Ou F-S, Venook AP, Hochster HS, Niedzwiecki D, Goldberg RM, et al. Impact of consensus molecular subtype on survival in patients with metastatic colorectal cancer: Results from CALGB/SWOG 80405 (alliance). J Clin Oncol. 2019;37(22):1876-85.

14. Stintzing S, Wirapati P, Lenz H-J, Neureiter D, Fischer von Weikersthal L, Decker T, et al. Consensus molecular subgroups (CMS) of colorectal cancer (CRC) and first-line efficacy of FOLFIRI plus cetuximab or bevacizumab in the FIRE3 (AIO KRK-0306) trial. Ann Oncol. 2019;30(11):1796-803.

15. Perez-Villamil B, Romera-Lopez A, Hernandez-Prieto S, Lopez-Campos G, Calles A, Lopez-Asenjo J-A, et al. Colon cancer molecular subtypes identified by expression profiling and associated to stroma, mucinous type and different clinical behavior. BMC Cancer. 2012;12(1):260.

16. Oh SC, Park Y-Y, Park ES, Lim JY, Kim SM, Kim S-B, et al. Prognostic gene expression signature associated with two molecularly distinct subtypes of colorectal cancer. Gut. 2012;61(9):1291-8. 
17. Sadanandam A, Lyssiotis CA, Homicsko K, Collisson EA, Gibb WJ, Wullschleger S, et al. A colorectal cancer classification system that associates cellular phenotype and responses to therapy. Nat Med. 2013;19(5):619-25.

18. De Sousa E Melo F, Wang X, Jansen M, Fessler E, Trinh A, de Rooij LPMH, et al. Poor-prognosis colon cancer is defined by a molecularly distinct subtype and develops from serrated precursor lesions. Nat Med. 2013;19(5):614-8.

19. Fontana E, Eason K, Cervantes A, Salazar R, Sadanandam A. Context matters-consensus molecular subtypes of colorectal cancer as biomarkers for clinical trials. Ann Oncol. 2019;30(4):520-7.

20. Morris JS, Luthra R, Liu Y, Duose DY, Lee W, Reddy NG, et al. Development and validation of a gene signature classifier for consensus molecular subtyping of colorectal carcinoma in a CLIA-certified setting. Clin Cancer Res. 2021;27(1):120-30.

21. Song N, Pogue-Geile KL, Gavin PG, Yothers G, Kim SR, Johnson NL, et al. Clinical outcome from oxaliplatin treatment in stage II/III colon cancer according to intrinsic subtypes: Secondary analysis of NSABP C-07/NRG oncology randomized clinical trial: Secondary analysis of NSABP C-07/NRG oncology randomized clinical trial. JAMA Oncol. 2016;2(9):1162-9.

\section{Figures}




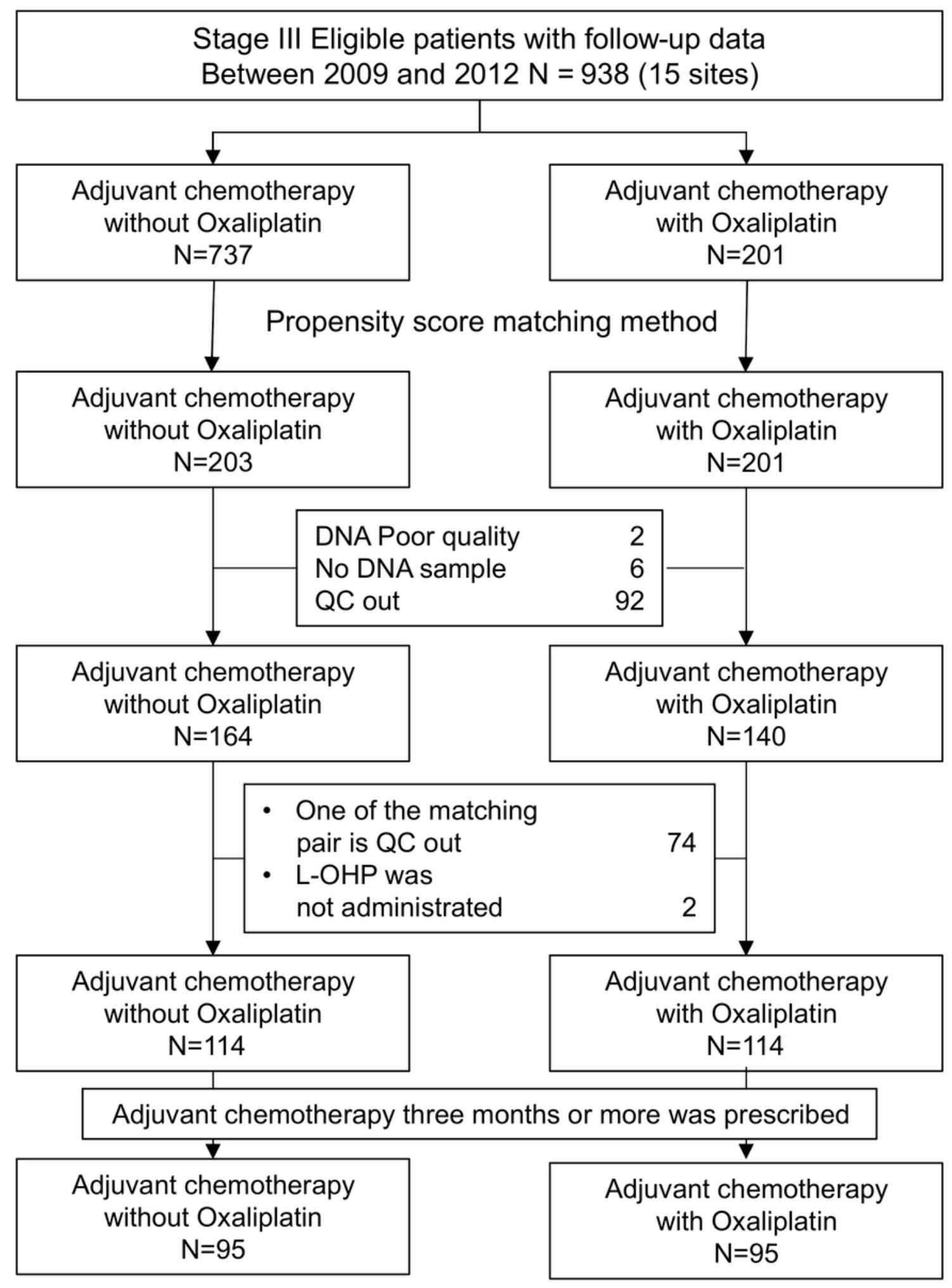

Figure 1

CONSORT diagram 


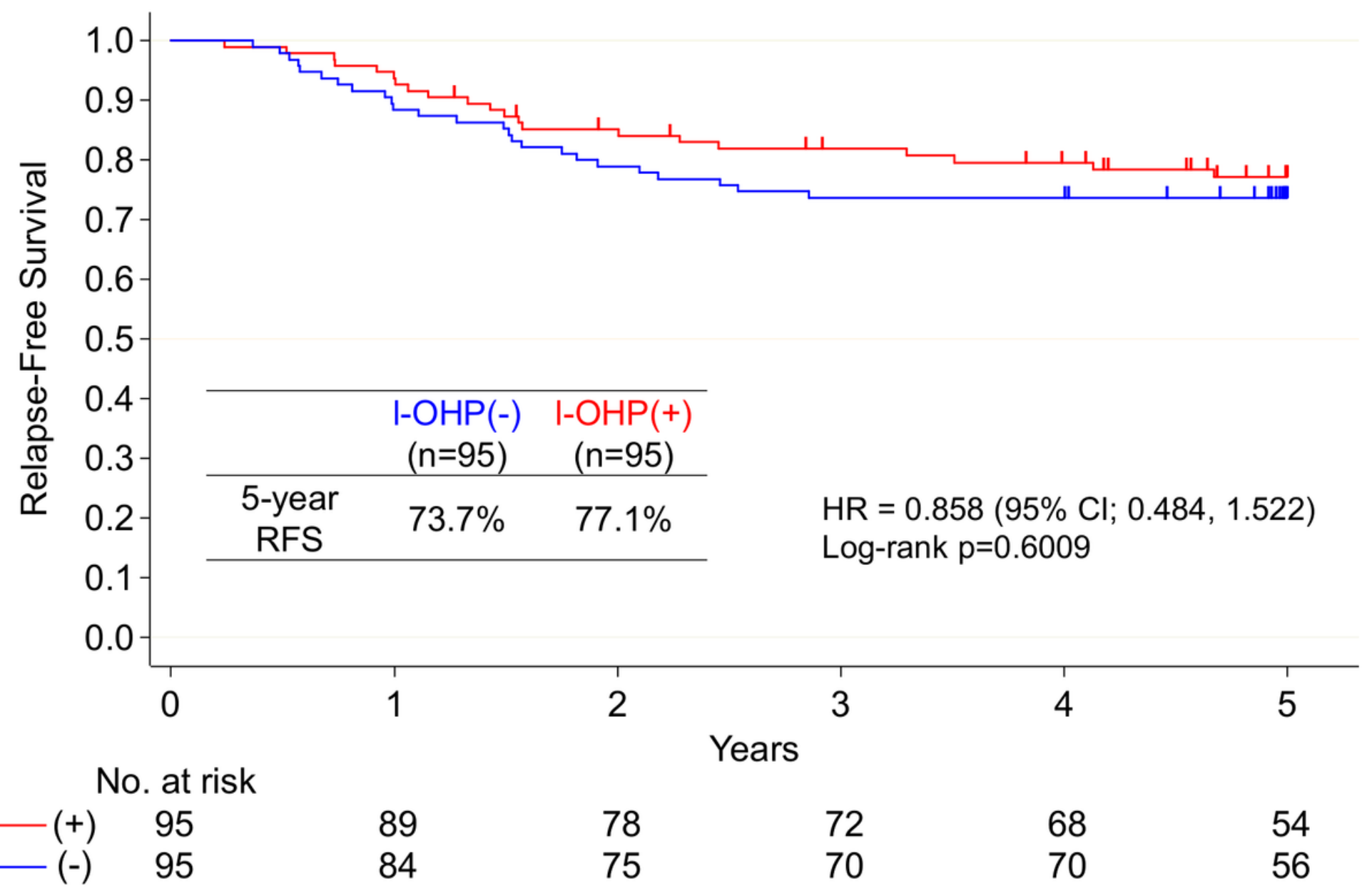

Figure 2

Five-year relapse-free survival (RFS) curves of all patients treated with [l-OHP(+), indicated in red] and without oxaliplatin [I-OHP(-), indicated in blue]. 
MSI-like, $n=52$

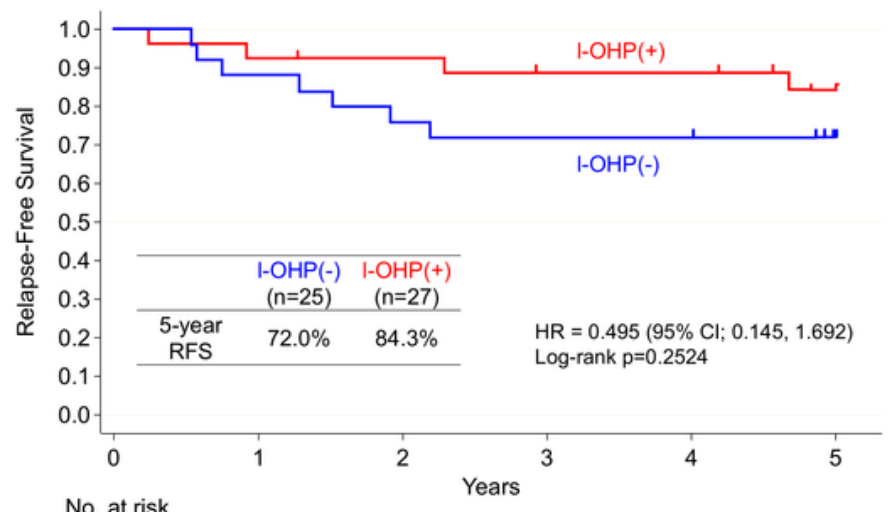

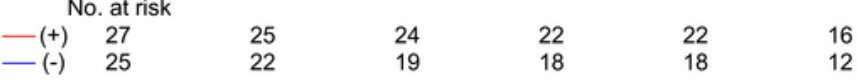

CIN-like, $n=78$

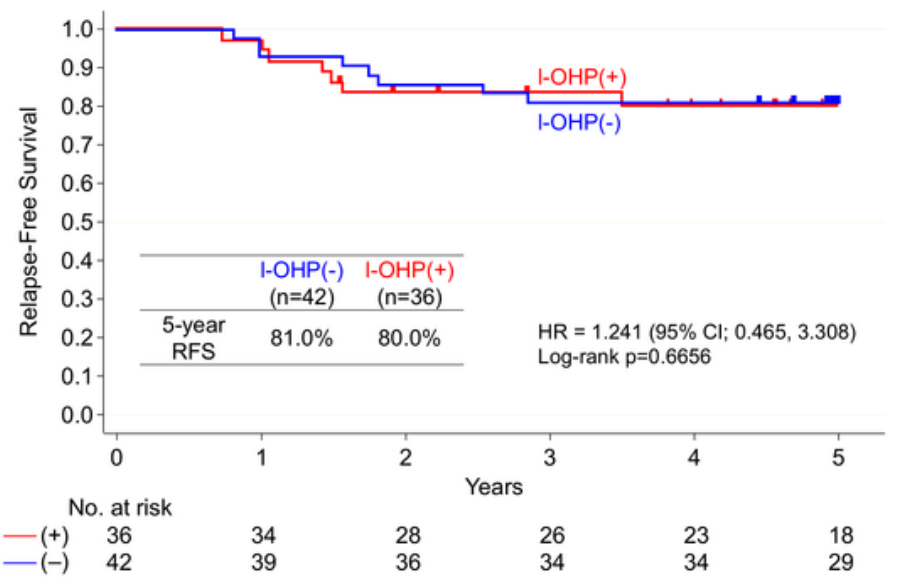

Stromal, $n=60$

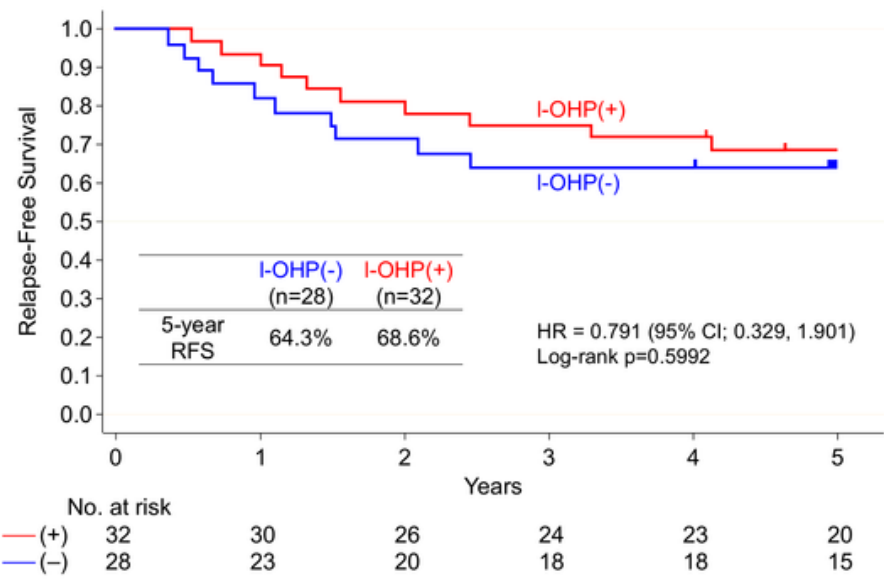

Figure 3

Five-year relapse-free survival (RFS) curves of patients treated with [I-OHP(+), indicated in red] and without oxaliplatin [I-OHP(-), indicated in blue] according to 55-gene classifier (55GC) subtypes [top: microsatellite instability (MSI)-like; middle: chromosomal instability (CIN)-like; bottom: stromal]. 

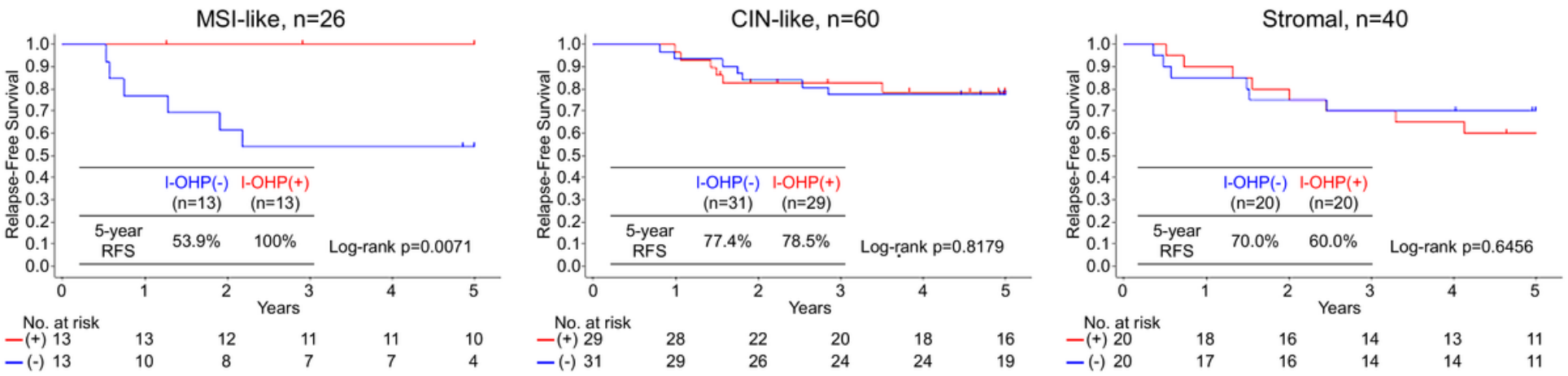

Right-sided tumor
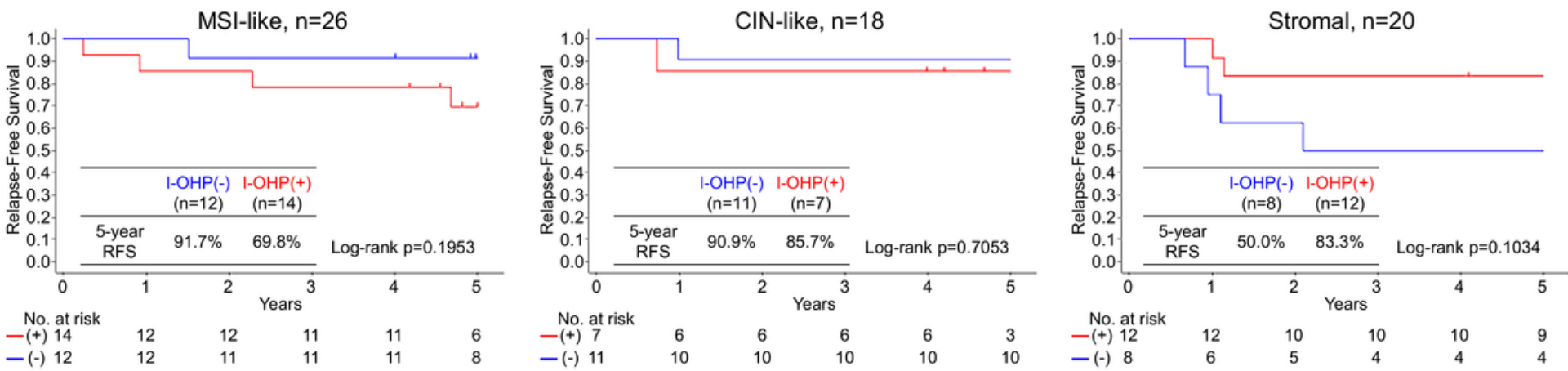

Figure 4

Five-year relapse-free survival (RFS) curves of patients treated with [l-OHP(+), indicated in red] and without oxaliplatin [I-OHP(-), indicated in blue] according to 55-gene classifier (55GC) subtypes and primary tumor sidedness (top: left-side; bottom: right-side).

\section{Supplementary Files}

This is a list of supplementary files associated with this preprint. Click to download.

- AdditionalFile.docx 